\title{
Design of Intelligent Access Control System Based on UWB Location Algorithm
}

\author{
MENG Xiang \\ Electrical \& Information Engineering College \\ Beihua University \\ Jilin, China \\ 731856651@qq.com
}

\author{
ZHAO Ying \\ Electrical \& Information Engineering College \\ Beihua University \\ Jilin, China \\ Zhaoying_jl@126.com
}

\begin{abstract}
The intelligent access control system based on UWB is designed in this paper. The system includes access controller, UWB reader array, electronic lock, power module, video capture modular and master computer. The access controller obtains the card's information by UWB reader array, the staffs' position can be located by UWB location algorithm. By comparing with information obtained by video capture modular, the system can indicates that if there is illegal invader. The location accuracy can reach $0.5 \mathrm{~m}$ and can identify accurately the invader's position. So the system can used widely in the place which requires fast walk.
\end{abstract}

Keywords- access controller;UWB reader array;UWB location algorithm; video capture modular

\section{INTRODUCTION}

With the continuous development and improvement of people's sense of safety and prevention, access control system is more and more widely used in modern office and house. The access control system in market has much disadvantage including structure and function's flaw and inefficiency. So the design of a responsive, powerful, easy to expand intelligent access control system is very important. The intelligent access control system based on UWB is designed in this paper. The system includes access controller, UWB reader array, door magnetism, electronic lock, power module, video capture modular, host computer and master computer. The system has higher location accuracy comparing with other access control system in market.

\section{UWB LOCATION ALGORITHM}

UWB(Ultra-Wide Band) location algorithm is used in this system because the algorithm has high location accuracy. Its principle is that the measured point sends a signal to the base stations of all reference points, the coordinate can be calculated by the arrival time of signal. According to the geometry principle of location, there will be 3 unknown number if we want to locate a measurement point's three-dimensional coordinate. The signal's time of arrival(TOA) is also unknown, so there are at least 4 reference points, 4 equations should be constructed in order to calculate the solutions. The equation is shown as follows: $\sqrt{\left(x-x_{i}\right)^{2}+\left(y-y_{i}\right)^{2}+\left(z-z_{i}\right)^{2}}=c\left(t_{i}-t_{0}\right)$

$i=1,2,3,4$

In the equation, $(\mathrm{x}, \mathrm{y}, \mathrm{z})$ is the location position's coordinate; $\left(\mathrm{x}_{\mathrm{i}}, \mathrm{y}_{\mathrm{i}}, \mathrm{z}_{\mathrm{i}}\right)$ is reference point's coordinate; $\mathrm{t}_{0}$ is the initial time of sending signal; $t_{i}$ is signal's arrival time. The measurement point's coordinate can be known if the equation can be solved.

The direct calculation method and the iteration method based on optimization can be used to solve the equation. In the direct calculation method, using time difference, after the substitution, the equations are simplified to obtain solutions. Usually the equations have two solutions and only a desired solution. If the calculated solution has no physical meaning or exceed the measuring range, the solution is regarded as invalid solution. If the two solutions are similar to, then their intermediate value should be taken.

The iteration method based on optimization is that the target function of the location coordinate is written as follows according to the equation(1).

$$
f(p)=\sum_{i=1}^{k}\left(\sqrt{\left(x-x_{i}\right)^{2}+\left(y-y_{i}\right)^{2}+\left(z-z_{i}\right)^{2}}-r_{i}\right)^{2}
$$

In formula(1), $\mathrm{k}$ is the number of reference points; ri is the distance between $\mathrm{i}$ reference point and target point; $p=[x, y, z]^{T}$ is coordinate of location point. The purpose of optimization is to find the minimum value of target function. The algorithm is shown as follows.

Step1:set a initial point $\mathrm{P}_{0}$, a initial matrix $\mathrm{B} 0$, let $\mathrm{k}=0$;

Step2:calculate $\mathrm{g} 0(\mathrm{~g}$ is gradient of targent function). $g_{k}=\nabla f(p)=\left[\frac{\partial f}{\partial x}, \frac{\partial f}{\partial y}, \frac{\partial f}{\partial z}\right]_{P=P_{k}}^{T}$;

Step3:determine the step size ak by precision onedimension search,

$$
\begin{aligned}
& f\left(P_{k}+a_{k} s_{k}\right)=\min f\left(P_{k}+a_{k} s_{k}\right), a_{k} \geqslant 0, \quad s_{k}=-B_{k} g_{k} \\
& \text { Step4:let } P_{k+1}=P_{k}+a_{k} s_{k} \text {,if }\left\|g_{k+1}\right\| \leqslant \varepsilon \text {, then } P^{*}=P_{k+1} \text {;if } \\
& \text { not, let } s_{k}=P_{k+1}-P_{k}, \quad y_{k}=g_{k+1}-g_{k} \text {,obtain Bk+1 by }
\end{aligned}
$$


correction

$$
B_{k+1}=B_{k}-\left(B_{k} y_{k} y_{k}^{T} B_{k}\right) / y_{k}^{T} B_{k} y_{k}+s_{k} s_{k}^{T} / y_{k}^{T} s_{k}
$$

formula

Step5:let $\mathrm{k}=\mathrm{k}+1$,if $\mathrm{k}<$ set value, then return step2;

Step6: Exit the loop, then output results.

Matlab program of the iteration method's error analyzing is shown as follows.

light_speed $=3 \mathrm{e} 8$;

$\mathrm{AP}=[0$ 0; 0 20; 20 0;20 20];

num_ap = length(AP);

Tag $=[1511]$

num_bits $=1000$;

ts $=1 / 20 \mathrm{e} 9$;

$\mathrm{k}=0$;

for $\mathrm{EbNo}=-40: 5:-30$

$\mathrm{k}=\mathrm{k}+1$;

for nloop $=1: 1$

TOA_tag $=($ length $($ sig $)-$ delay 1$) *$ ts;

TOA_1 = (TOA_ap1 + TOA_tag) $/ 2$;

TOA 2 = Td ap1 tag2 - TOA 1 + TOA tag;

TOA_3 = Td_ap1_tag3 - TOA_1 + TOA_tag;

time_dur = [TOA_1 TOA_2 TOA_3]; end

toa_error(k,nloop) $=$ toa(AP,Tag,time_dur,light_speed)

end

plot(-15:5:0, toa_error);

The comparison chart of the direct calculation method and the iteration method's location accuracy is shown in Figure 1.

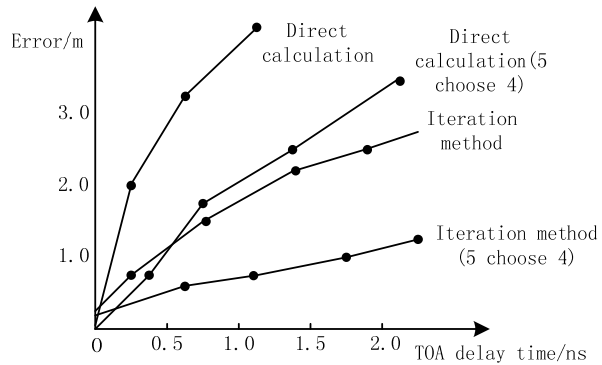

Figure1. The comparison chart of the direct calculation method and the iteration method's location accuracy

The TOA of signal from the measurement point to the basic station obey $N\left(0, \delta^{2}\right)$ distribution. The repeated measurement average time will close to the true value, so the time averaging method is used to improve the location accuracy. The TOA of 10 times is averaged at the base of iteration method ( 5 choose 4 ), the location accuracy can reach $0.6 \mathrm{~m}$. The method can improve $25 \%$ comparing with the normal iteration method. The location accuracy is higher if averaging times increase. The accuracy comparison chart of different number for time-average is shown in Figure 2.

Figure 2 shows that the location accuracy can reach $0.2 \mathrm{~m}$, but the price is the increasing of measurement number, the location time and the power consumption., so average time of 20 times is used in this paper.

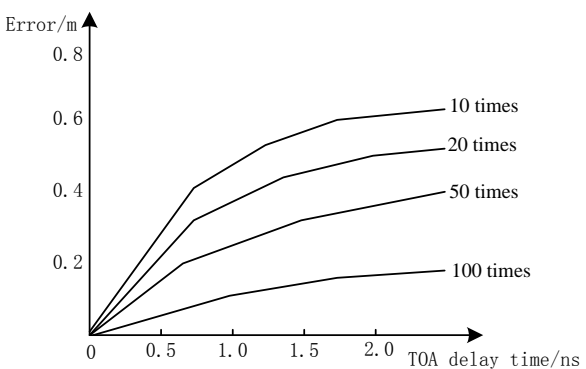

Figure2. The accuracy comparison chart of different number for timeaverage

\section{DESIGN OF HARDWARE}

The diagram of access controller's hardware circuit is shown in Figure 3. The system includes access controller, UWB reader array, door magnetism, electronic lock, power module, video capture modular, host computer and master computer. ARM chip S3C6410 is chosen as the main microcomputer because it had better function and fast performance speed. First, access controller initializes external equipments, including UWB reader array, communication, door magnetism, database and so on. Then access controller goes into monitoring state, once detects the card to be present, the controller reads card's information by UWB reader array. The card's information includes ID, position, number and so on. The card's number is the legal stuff's number. By comparing with information obtained by video capture modular, the system can indicates that if there is illegal invader. If there is illegal invader, the controller locates the invader's position by UWB location algorithm, and marks the position of the invader at the video image, and at the same time the controller gives alarm. The relevant information will be saved in the local and remote database.

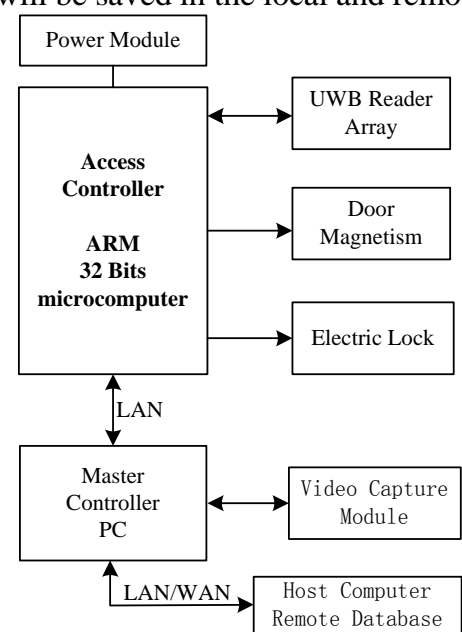

Figure 3. The diagram of access controller's hardware circuit

In the access control system, controller communicates with the master computer by LAN network. Controller sends the information obtained to the master computer and at the same time executes the dynamic command received. The master computer can connect with remote database by 
TCP/IP protocol. The access controller and the UWB reader array communicate by RS485 bus. JTAG interface is mainly used to test inner device, emulation and debug, and it is a simple and effective method of develop and debug. The JTAG interface's connection circuit with S3C2440 is shown in Figure 4.

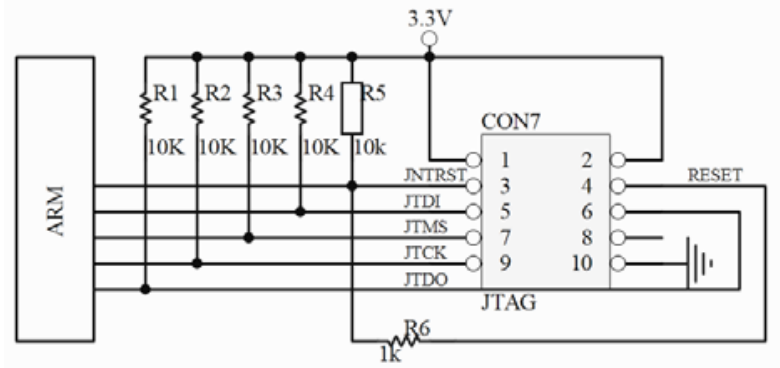

Figure4. JTAG interface's connection circuit with ARM

ARM chip S3C6410 is chosen as the main microcomputer, the diagram of main microcomputer is shown in Figure 5. 256M DDR memory and 256M Flash memory are expand, and at the same time $2 \mathrm{COM}$ interface and 1 LAN interface are designed. S3C6410 has ARM 1176TEF-S kernel, its highest frequency can reach $667 \mathrm{MHz}$, so it can completely satisfy the requirement of access control system at speed.

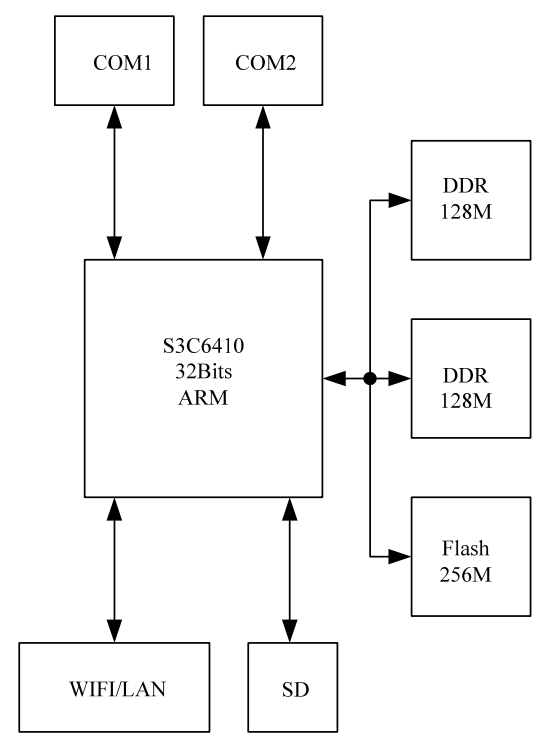

Figure5. The diagram of main microcomputer

\section{DESIGN OF SOFTWARE}

The access controller's flow diagram of main program is shown in Figure 6.

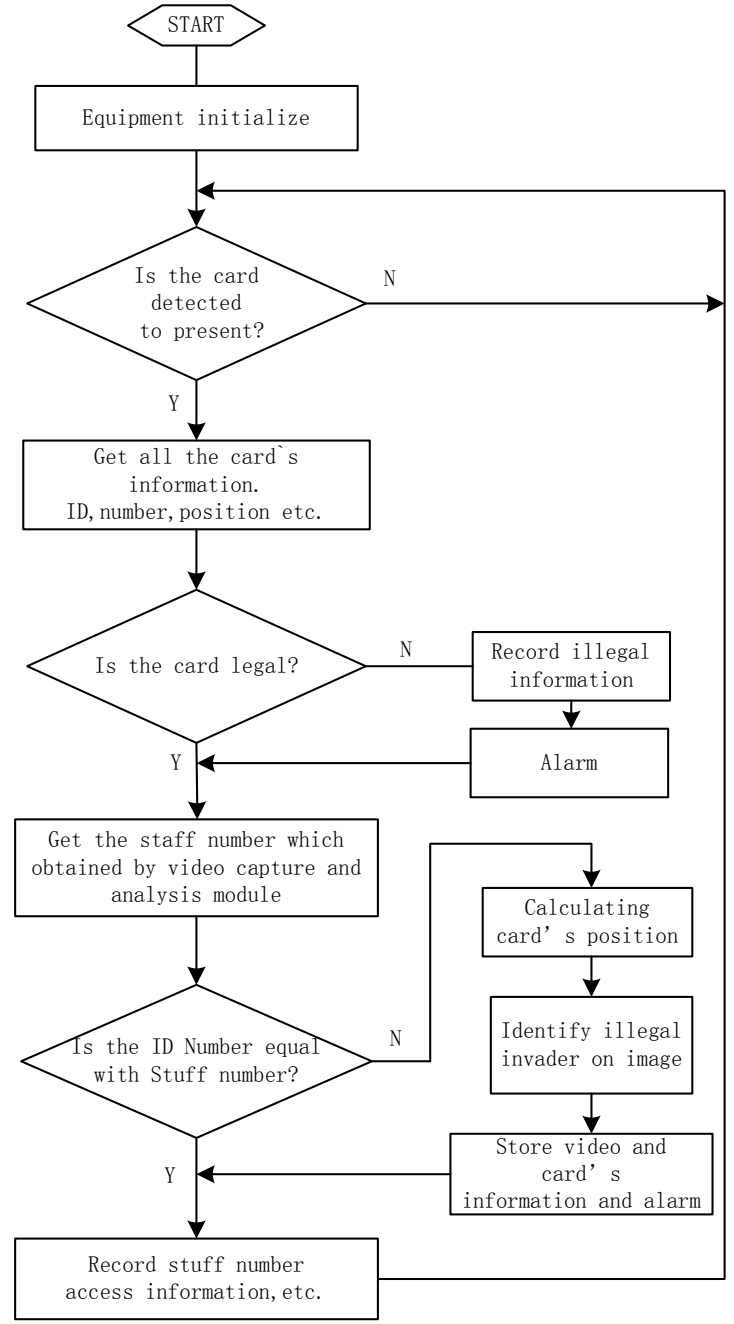

Figure 6. The access controller's flow diagram of main program

\section{CONCLUSION}

The intelligent access control system is designed in this paper. The system includes access controller, UWB reader array, door magnetism, electronic lock, power module, video capture modular, host computer and master computer. Then access controller reads card's information by UWB reader array. By comparing with information obtained by video capture modular, the system can indicates that if there is illegal invader. If there is illegal invader, the controller locates the invader's position by UWB location algorithm, and marks the position of the invader at the video image, and at the same time the controller gives alarm. UWB(UltraWide Band) location algorithm is used in this system and the location accuracy can obtain $0.5 \mathrm{~m}$. The hardware diagram and corresponding software flow diagram are given in detail. The system choose ARM chip S3C2440 as main microcomputer, so the system had faster performance and better stability. The master computer and the access controller communicate by LAN network. The computer 
managerial interface is simple and operates easily. So the system has extensive application prospect.

\section{ACKNOWLEDGMENT}

The paper is sponsored by Fund project of Jilin Province Education Office (Disordered detection intelligent access control system based on RFID technology and Image recognition).

\section{REFERENCES}

[1] CAO Chengmao,LI Yujie and MA Degui, "Design of intelligent measuing force apparatus and how to improve its accuracy,”ISTM/2009,2009,pp.459-460.

[2] XIE YA-qin,ZHANG Ye-rong, "TOA Based UWB Location Algorithms, "Journal of Nanjing University of Posts and telecommunications , vol.27,2011,pp.73-76.
[3] Pang Yan, ZHANG Li-jun, "An Improved Algorithm for UWB Precision Positioning Based on Time Averaging, ”Journal of Beijing Jiaotong University, vol.29,2010,pp.60-63.

[4] ARM920T(Rev1)Technical Reference Manual,ARM Limited,2010,pp.55-60.

[5] XU Xi, "Study of embedeed communication system based on internet,” Master degree paper of Guizhou university,2008,pp.45-48.

[6] DU Qifei, "Development and study of network intelligent instrument based ARM,"Master degree paper of Taijin university,2009,pp.45-48.

[7] .LI shanping and LIU Wenfeng, "Linux and embedeed system”,publisher of Tsinghua university,2009,pp.30-35.

[8] YU Lu, " Hardware design of embeded system based on ARM,”Master degree paper of Zhejiang university,2010,pp.85-88.

[9] KEGEN Y,LAN O, "Performance of UWB position estimation based on time-of-arrival measurements,"Proc of IEEE ultra wide band conference,2011,pp.400-404.

[10] CHAN YT,HO KC, "A simpal and efficient estimator for hyperbolic location, "IEEE transactions on signal processing,2010,pp.302-305. 